\title{
Foundations for Cyber Zone Defense
}

\author{
Robert Mitchell \\ Sandia National Laboratories \\ Albuquerque, NM 87185 \\ Email: rrmitch@sandia.gov
}

\author{
Paul Sery \\ Sandia National Laboratories \\ Albuquerque, NM 87185 \\ Email: pgsery@sandia.gov
}

\author{
Tom Klitsner \\ Sandia National Laboratories \\ Albuquerque, NM 87185 \\ Email: tklitsn@sandia.gov
}

\begin{abstract}
We will introduce a new framework called cyber zone defense (CZD) that treats malware like a black box: a process we can study solely based on its internal and external communication. We can reduce the impact of malware, without regard to its functionality or even existence, by limiting only these connections. In this paper, we propose two metrics for measuring CZD effectiveness and an illustrative simulation and a closed form mathematical model that predicts these statistics. The simulation is intuitive; it allows the analyst to provision arbitrary configurations and see how changes in topology affect the efficacy of the CZD. The model provides a mathematical verification for CZD and matches the results of the simulation well. These artifacts test the feasibility of CZD while deferring implementation details.
\end{abstract}

\section{INTRODUCTION}

Cyber security is a critical topic: sophisticated cyber attackers are motivated by power and money, and the systems they target are growing in complexity [1], [2]. The cyber world is becoming more complex, and complexity gives the attacker an advantage over the defender.

Intrusion prevention, detection, tolerance and response is a widely used conceptual model for cyber defense; it is the counterpart to an attack model. Cyber zone defense (CZD) [14] is the umbrella for techniques aiming to render cyber attacks inert by limiting the attacker visibility, access, lateral movement and reachback. From a tactical perspective, CZD accomplishes intrusion prevention by reducing the attack surface area in a network. In kinetic warfare, security forces work to channelize their adversary. For example, they do this artificially with entry control points and organically by occupying key terrain. By channelizing the attacker, the defender chooses the location of an encounter and concentrates defensive resources. In cyber warfare, we seek to do the same by driving adversaries to well-known entry and exit points where our visibility and control mechanisms are at their best. However, CZD is most effective against command and control (C2) and exfiltration. From a strategic perspective, CZD accomplishes intrusion tolerance by limiting internal and external communication. Again, there are kinetic warfare parallels for this. Maritime warfighters segment ships into many isolatable compartments. If one compartment is

Sandia National Laboratories is a multi-program laboratory managed and operated by Sandia Corporation, a wholly owned subsidiary of Lockheed Martin Corporation, for the U.S. Department of Energy's National Nuclear Security Administration under contract DE-AC04-94AL85000. Approved for unlimited release: SAND2016-1624 C. compromised, the impact is localized and the ship continues to fight Also, when dealing with a belligerent, security forces may locally disable communication networks in order to deny the malfeasant the ability to receive guidance from their command element. CZD aims to isolate potential cyber threats and render them inert. Cyber defenders spend a lot of effort protecting networks and devices against specific threats. For instance, intrusion detection systems look for anomalies and signatures indicating an attack. While this methodology is and will remain the foundation of our security infrastructure, it is fundamentally reactive and will remain a step behind in the increasingly inhospitable threat environment. The solution is to consider human adversaries and their malware as a black box whose inside we cannot visualize. We concede that bad actors will implant malware on our network somehow but we aim to neutralize them by limiting or eliminating their internal and external communication. It should not matter how malware is introduced or how it works if it cannot communicate outside of the victim. Thus, the black box concept provides a powerful tool that is independent of the functionality and organization of malware. In order to test the efficacy of CZD we propose the following attack model.

We model the adversary using the six phase attack sequence comprising: survey, tool, implant, pivot, damage/exfiltrate and cleanup stages; Figure 1 illustrates this progression. During the survey phase, the attacker identifies the key locations for the attack: the vulnerable node (e.g., web server or operator workstation) through which to enter the defender's system, the critical nodes [e.g., domain controller or supervisory control and data acquisition (SCADA) programmable logic controller (PLC)] and the intermediate nodes linking the entry node and critical nodes. Survey data may include host name, subnet, network address, media access control (MAC) address, operating system and security and application software. During the tool phase, the attacker configures existing attack tools or creates new tools. During the implant phase, the attacker establishes a presence on the defender's system. This could be from attacking a webserver, phishing an unwitting human operator or tasking a witting insider. During the pivot phase, the attacker will transition from the entry node to the critical node. During a damage phase, the attacker will perform some application specific action to disrupt the defender's core mission. Alternatively, during an exfiltrate phase, the attacker will steal the defender's critical data. During the cleanup phase, the attacker will remove all artifacts from the attack 


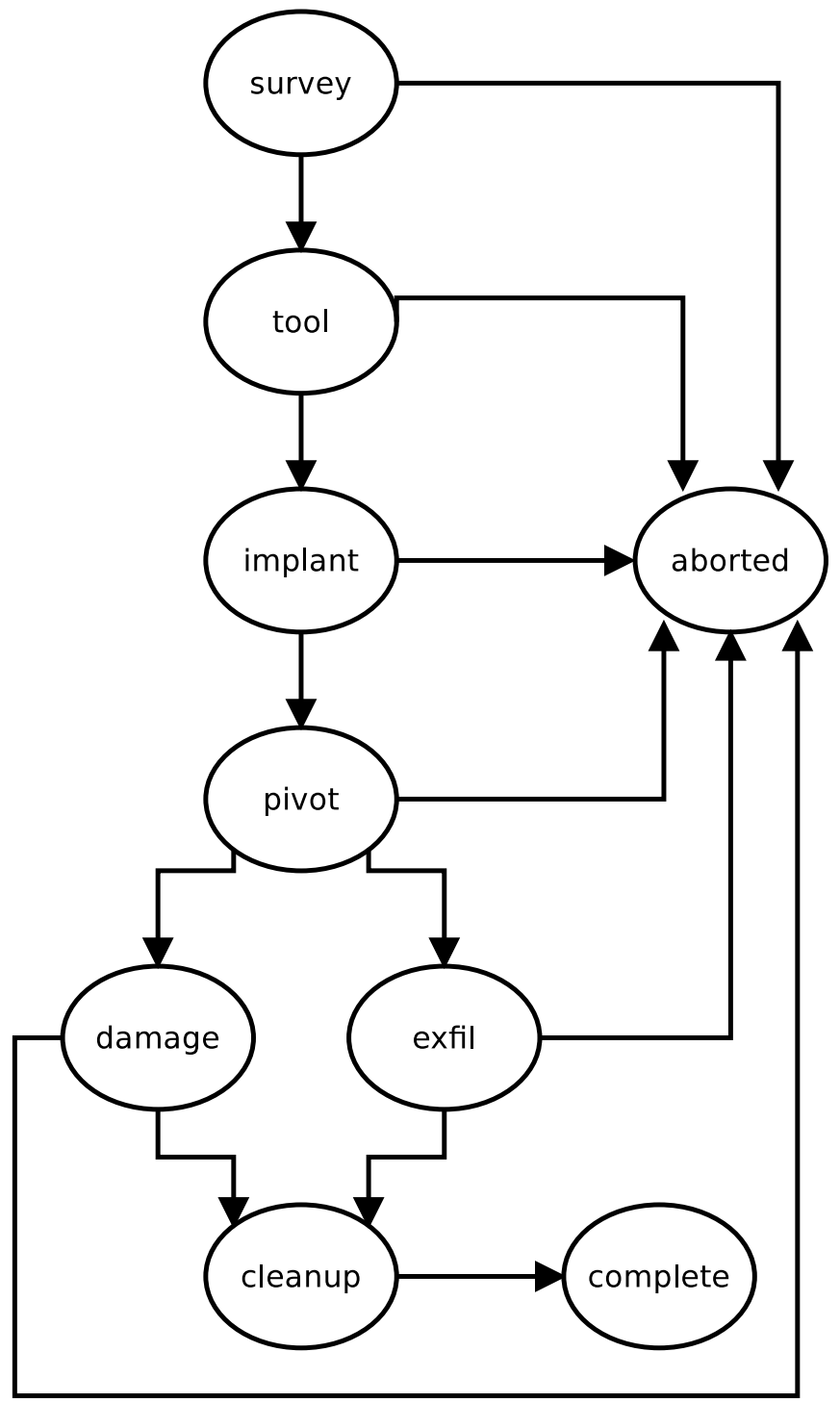

Fig. 1. Phased attack sequence.

(e.g., registry entries, covert file systems and tainted binaries). In this study, we propose a way to quantify the effectiveness of CZD. We consider a highly simplified configuration in order to provide a solid foundation for future work that will approach a realistic level of sophistication and fidelity.

\section{LiteraturE SEARCH}

Our literature search revealed that there is a gap in the literature with respect to predictive modeling applicable to CZD performance. Many studies lack numerical data, and those that have it often use metrics that measure the impact to the defender rather than the attacker. We fill this gap by modeling probabilities of compromise and reachback.

The following papers propose interesting approaches to CZD, but do not provide any numerical results: Lai and Tai [10] survey a number of ways to instrument network isolation that fit into the framework of the ISO-17799 standard. Wantian and Chengzhi [15] investigate the effects of network isolation on a smart grid. Yu, et al. [16] study the effects of network isolation on a manufacturing environment. Conklin [3] intends to isolate a SCADA system based on system state rather than network traffic. To accomplish this, the system changes state rather than exchanging network traffic. While this is not a viable approach to an enterprise network, our approach is because it simplifies the problem by identifying and constricting upstream chokepoints. Gong, et al. [7] investigate isolating applications from one another within a single host; on the other hand, our work considers isolating hosts from one another within a network.

The following papers provide numerical results that measure the impact to the defender rather than the attacker: Liu, et al. [12] study a two tier (cyber zone) approach to secure software defined networking (SDN): the tiers comprise the control and data planes. The authors measure the performance of their design in terms of load balance level, percentage of packet loss and simulation time. Costa and Costa [4] study isolation vulnerabilities and associated remedies for FlowVisor-based virtual networks. The authors measure the performance of their design in terms of communication throughput. Liang, et al. [11] investigate a MAC based approach to isolating virtual machines. The authors measure the performance of their design in terms of startup time and benchmarks. Cuiñas, et al. [5] study an innovative approach to network isolation: vegetation. The authors measure the performance of their design in terms of radio frequency (RF) attenuation and minimum and maximum separation. Fernandes, et al. [6] investigate using OpenFlow to isolate networks by using a different protocol stack for each segment. The authors measure the performance of their design in terms of received packet rate, round trip time, jitter and aggregated packet rate. Nunes, et al. [13] study a virtualized approach to network isolation, specifically tailored for cloud computing data centers. The authors measure the performance of their design in terms of network latency.

We found two contemporary papers that study the impact of cyber zone defense to the attacker: Khoury, et al. [9] research a potential drawback of network isolation: creating critical points in the topology that make the network prone to cascading failure. The authors measure the performance of their design in terms of robustness, post attack viability, saved nodes and percentage of dead networks saved. Han, et al. [8] investigate type based isolation (TBI) as a means for securing a subject network. Their traffic types comprise MSN, QQ, HTTP and file transfer protocol (FTP). The authors contrast TBI with IP based isolation (IPBI), exclusive IPBI and selective IPBI. Their basic concept is to isolate hosts reactively based on a positive intrusion detection. The authors instrument a 230 node simulation to generate their dataset which comprises counts of infected nodes and susceptible nodes.

\section{Simulation AND Model}

The simulation and model we propose predict two metrics: the probabilities of compromise and reachback. The probability of compromise calculates the likelihood that the 
attacker has implanted an arbitrary host. The probability of reachback calculates the likelihood that an arbitrary host is compromised and able to connnect to a $\mathrm{C} 2$ server to exfiltrate victim data or receive tasking. While probability of compromise is an intuitive metric, probability of reachback is a novel measurement of the attacker's effectiveness: Malware needs to receive remote tasking and exfiltrate victim data. In this paper, we assume an insider threat: the adversary has compromised one node. However, this work can be extended to begin with an outsider threat. This is a minor modification; when an outsider authenticates and escalates privilege, they are indistinguishable from an insider.

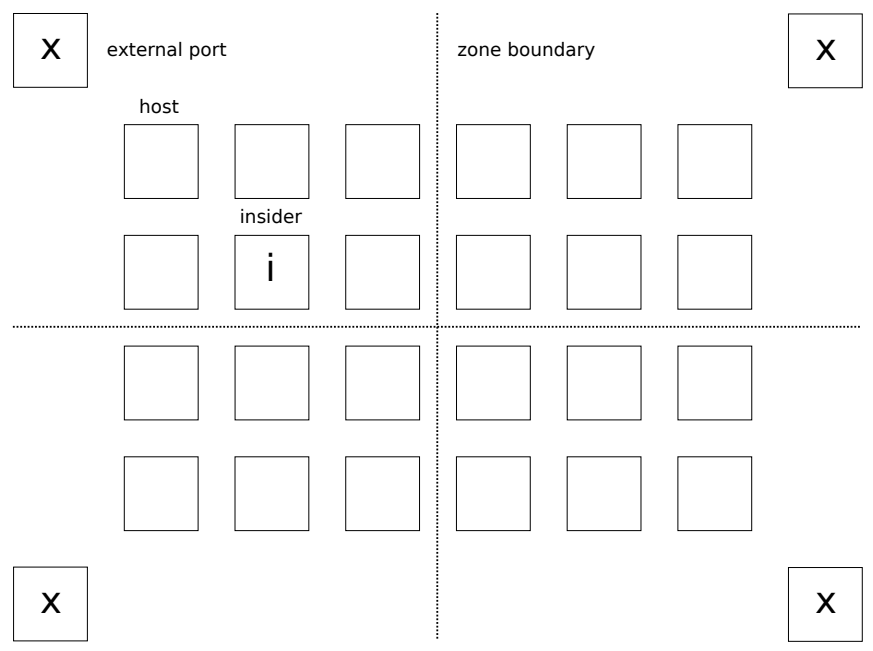

Fig. 2. Simplified example network.

Figure 2 illustrates a basic 28 node network with four zones, four external ports and one insider.

Our simulation and model are based on two basic probability concepts. First, the joint probability of two independent events is the product of their respective probabilities.

$$
P(A \cap B)=P(A) \cdot P(B)
$$

Second, the probability of an event is one minus the probability of the event's complement.

$$
P(\neg A)=1-P(A)
$$

\section{A. Simulation}

Our simulation iteratively calculates the probability each node in the network is compromised; during each round, the malware attempts to push one node farther away from previously compromised hosts. During each round, the simulation calculates new probabilities of compromise based on the probability an exploit exists, the network topology and the results of the previous round. Specifically, if the node's probability of compromise is zero, the simulation recalculates the probability of compromise for the node based on the probability an exploit exists, the network topology, the node identifier and the results of the previous round. To calculate the probability of compromise for a node, the simulation
TABLE I

SIMULATION PARAMETERS.

\begin{tabular}{|l|l|l|}
\hline \multicolumn{1}{|c|}{$\begin{array}{c}\text { Parameter } \\
\text { Name }\end{array}$} & \multicolumn{1}{c|}{ Description } & \multicolumn{1}{c|}{ Source } \\
\hline \hline$p_{c}$ & probability of compromise & Simulation \\
\hline$p_{r}$ & probability of reachback & Simulation \\
\hline external ports & Internet facing ports & Defender \\
\hline neighbors & network adjacencies & Defender \\
\hline insiders & initial insiders & Attacker \\
\hline$p_{e}$ & probability an exploit exists & Attacker \\
\hline
\end{tabular}

calculates the probability that none of the node's neighbors can implant it. This is equivalent to the product of the probabilities that each of the node's neighbors cannot implant the node. The result is one minus this product. The probability a neighbor cannot implant is one minus the probability a neighbor can implant. The probability a neighbor can implant is the probability of compromise of the neighbor times the probability an exploit exists. The simulation iterates until the probabilities of compromise for the network converge. Figure 3 visualizes a simple three round simulation: an insider compromises a target of opportunity in round zero with probability one, pivots to an intermediate host in round one with probability 0.5 and gains access to the high value target in round two with probability 0.25 .

To calculate the probability of reachback, the simulation calculates the product of the probability of integrity for each external port. The result is one minus this product (that all external ports are not compromised). The probability of integrity is one minus the probability of compromise.

$p_{c}$ (probability of compromise) and $p_{r}$ (probability of reachback) are the outputs of our simulation. The analyst provides four parameters to the simulation. The defender chooses two: external ports (Internet facing ports) and neighbors (adjacencies for each node). The attacker chooses the other two: insiders (attacker's initial compromised hosts) and $p_{e}$ (probability an exploit is available for a given configuration). Because all software has vulnerabilities, $p_{e}$ is a function of the budget and/or skill level of the attacker. Table I summarizes these parameters

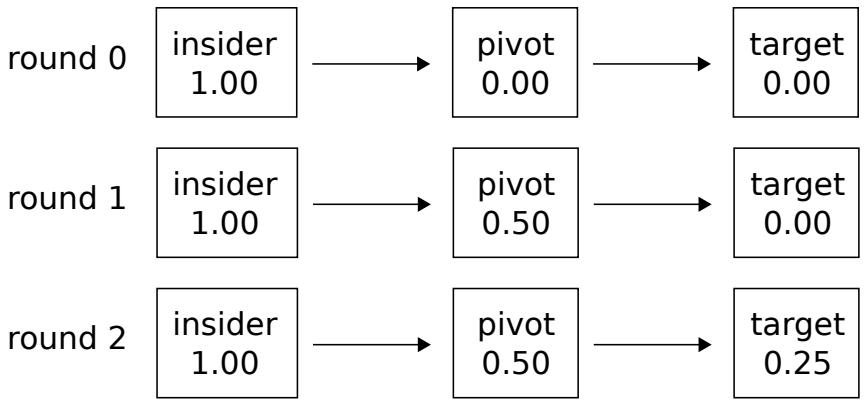

Fig. 3. Simple three round simulation. 


\section{B. Closed Form Mathematical Model}

In order for an arbitrary node to be compromised, the adversary must have an exploit for the node, and it must be in the same zone as the insider. First, the probability a node is in the same zone as the insider can be estimated by the number of nodes in a zone less one (to account for the arbitrary node) over the size of the network less one. Next, the probability an arbitrary node is compromised is the probability the adversary has an exploit for the node times the probability that it is in the same zone as the insider. The probability of the adversary having an exploit for an arbitrary node is a function of the attacker's budget and the defender's architecture. The attacker's budget comprises short term investment in skilled contractors and long term investment in grooming organic talent. On the other hand, the defender's architecture may be monocultural or demonstrate platform diversity. Finally, we adjust the probability of compromise to account for the fact that one node is known to be compromised: The adjusted probability of compromise is one plus the probability of compromise times the network size less one all over the network size. Essentially, this corrects nonintuitive results for small network and zone sizes. Equation 3 models the probability of compromise.

In order to reachback to the $\mathrm{C} 2$ server, a node with an external port (Internet connection) must be compromised. First, the probability a node is secure is one minus the probability of compromise. Next, the probability that no external port is compromised is the probability that an arbitrary node is secure raised to the number of external ports. Third, the probability an external port is compromised is one minus the probability that no external port is compromised. Finally, the probability of reachback is the probability an external port is compromised. Equation 4 models the probability of reachback.

$$
\begin{gathered}
p_{c}=\frac{1+p_{e} \cdot \frac{z-1}{n-1} \cdot(n-1)}{n} \\
=\frac{1+p_{e} \cdot(z-1)}{n} \\
p_{r}=1-\left(1-p_{c}\right)^{x}
\end{gathered}
$$

$p_{c}$ (probability of compromise) and $p_{r}$ (probability of reachback) are the outputs of our closed form mathematical model. Applying Equations 3 and 4 to the subsequent parameters yields $p_{c}$ and $p_{r}$, respectively. The analyst provides the following parameters to the model: $p_{e}$ indicates the probability an exploit is available for a given configuration. Because all software has vulnerabilities, $p_{e}$ is a function of the budget and/or skill level of the attacker. The defender chooses the rest of the parameters: $z$ (zone size), $n$ (network size) and $x$ (number of external ports). Table II summarizes these parameters.

\section{REsults}

In Figures 4 through 9, the points come from the simulation data set and the curves plot the mathematical model.
TABLE II

CLOSED FORM MATHEMATICAL MODEL PARAMETERS.

\begin{tabular}{|l|l|l|}
\hline $\begin{array}{c}\text { Parameter } \\
\text { Name }\end{array}$ & \multicolumn{1}{|c|}{ Description } & Source \\
\hline \hline$p_{c}$ & probability of compromise & Model \\
\hline$p_{r}$ & probability of reachback & Model \\
\hline$p_{e}$ & probability an exploit exists & Attacker \\
\hline$z$ & zone size & Defender \\
\hline$n$ & network size & Defender \\
\hline$x$ & number of external ports & Defender \\
\hline
\end{tabular}

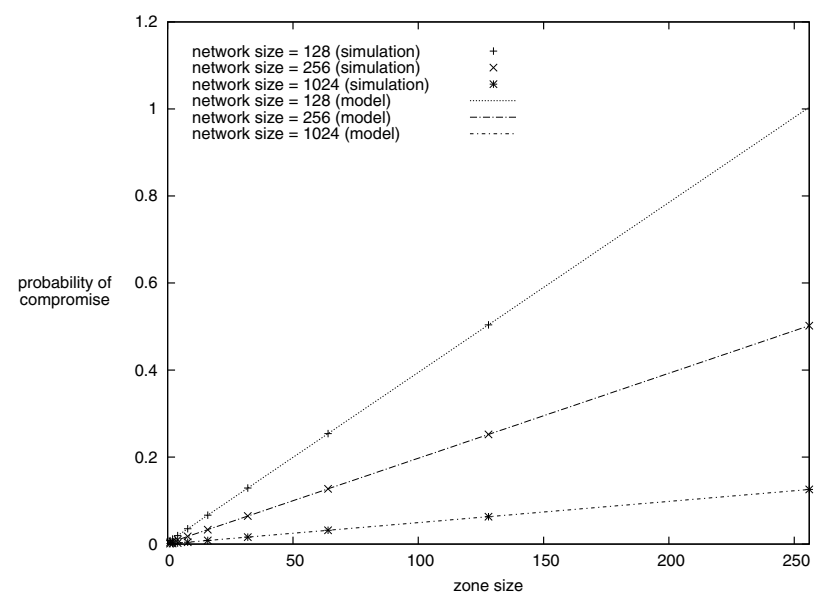

Fig. 4. Probability of compromise versus zone size and network size.

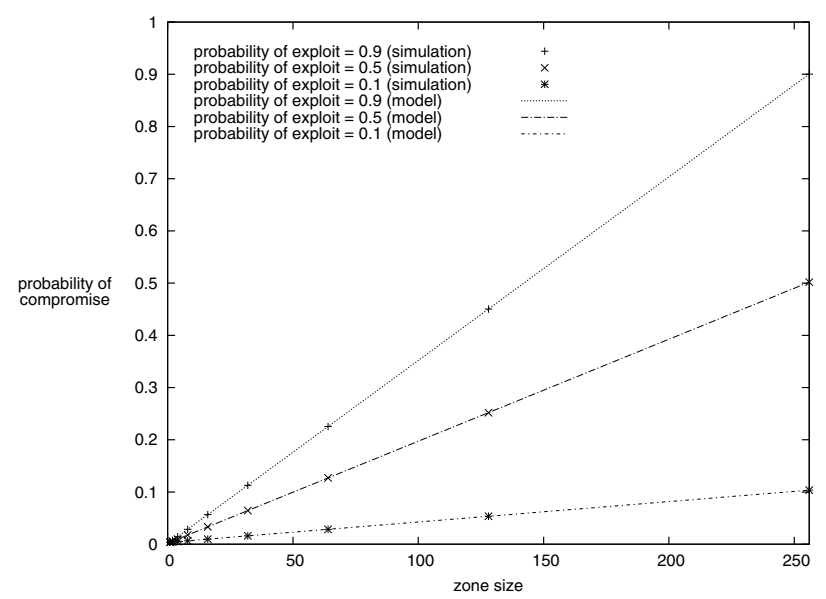

Fig. 5. Probability of compromise versus zone size and probability exploit exists.

The basic trends for Figures 4 through 6 are as expected: a linear relationship between zone size and probability of compromise. First, Figure 4 shows larger networks have a lower probability of compromise. This is because as the overall network size grows in relation to the IZ size, less nodes are candidates for infection; it is an amortization of $p_{c}$ across more nodes. Next, Figure 5 shows a direct relationship between 


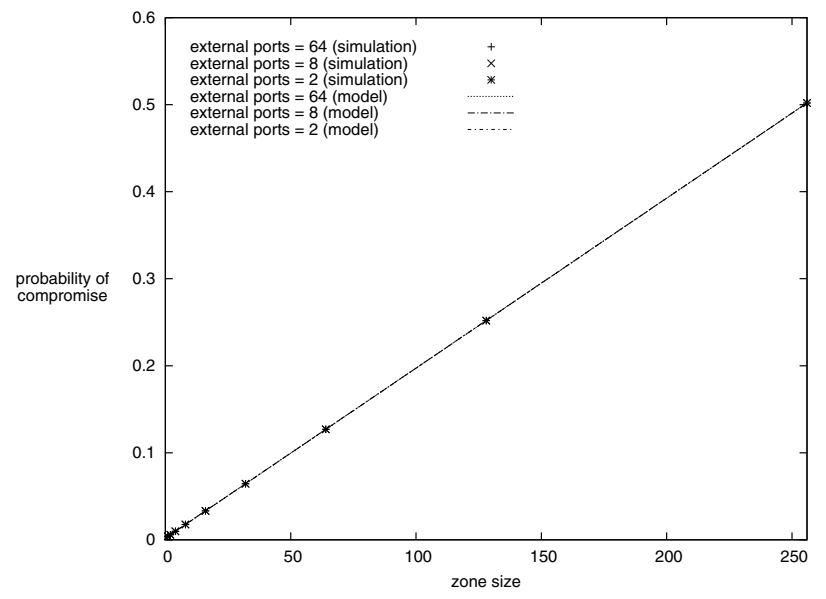

Fig. 6. Probability of compromise versus zone size and number of external ports.

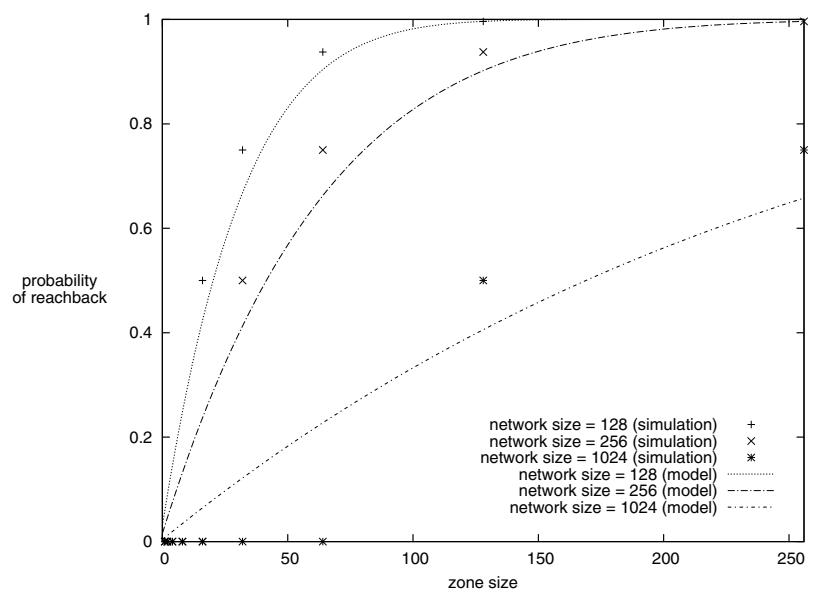

Fig. 7. Probability of reachback versus zone size and network size.

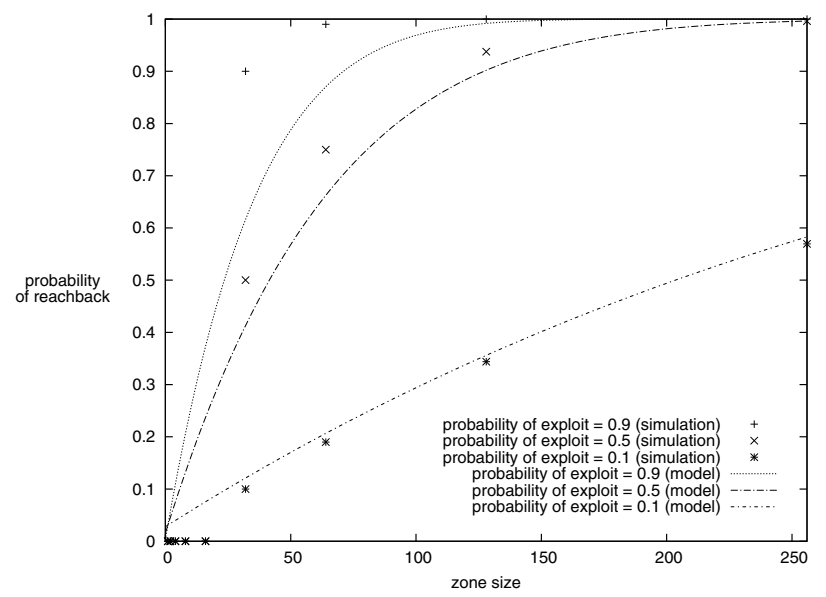

Fig. 8. Probability of reachback versus zone size and probability exploit exists.

probability of exploit and probability of compromise; this is because each host becomes more likely to be infected.

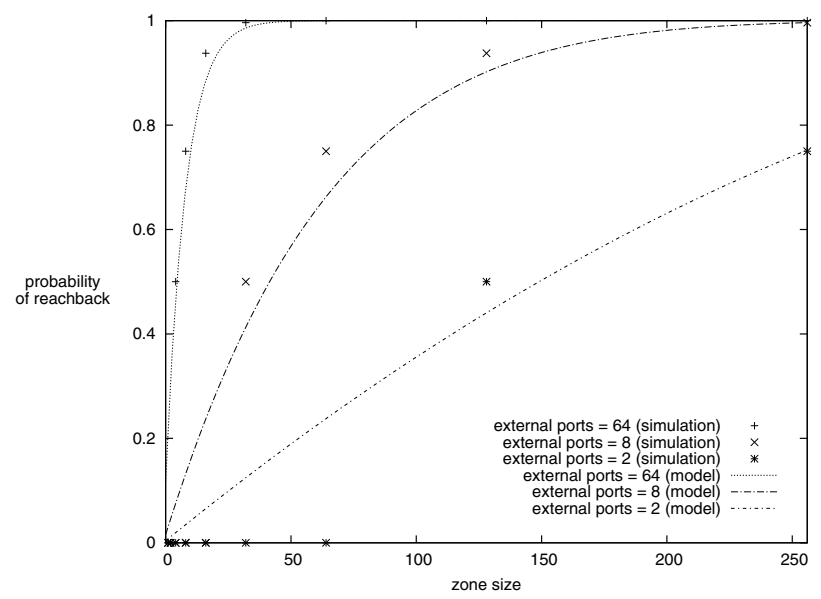

Fig. 9. Probability of reachback versus zone size and number of external ports.

Third, Figure 6 shows the number of external ports has no impact on the probability of compromise; this is because the number of external ports has no bearing on the probability of compromise. This figure is included for completeness.

The basic trends for Figures 7 through 9 are as expected: a sublinear, perhaps inverse exponential, relationship between zone size and probability of reachback. First, Figure 7 shows larger networks have a lower probability of reachback; this is because as the overall network size grows in relation to the IZ size, less external ports become candidates for infection. Next, Figure 8 shows a direct relationship between probability of exploit and probability of reachback; this is because each external port becomes more likely to be infected. Third, Figure 9 shows a direct relationship between the number of external ports and probability of reachback; this is because there are more candidate victims with Internet visibility.

The simulation points match the mathematical model curves very closely. While the probability of compromise results for the simulation and mathematical model match, the mathematical model provides a good approximation for the simulation probability of reachback. The mean squared error (MSE) between the simulation and mathematical model results is 0.021 for probability of reachback. Most of this error occurs when the zone size is near zero due to the strategy the simulation uses to position the external ports; in future work, we will shrink this error by randomizing the position of the external ports in the simulation and increasing the number of simulation runs.

Overall, as an IZ grows in size to match that of its host network, it becomes indistinguishable in behavior.

\section{CONCLUSions}

Our initial results indicate CZD is a viable cyber defense technique and should be studied further. We also recognize that the simulation and model presented here are starting points; we liken the simplifying assumptions in this paper with those made when learning the laws of motion in physics. 
Our future work will focus on simulation. Now that we have established some basic axioms in a simplified cyber system, we will add complications to the simulation to make it more robust. We will do this in four ways: First, iteration or time limits will capture the real world challenge of a dynamic attack surface. Phenomena that naturally create a dynamic attack surface are patch distribution, host obsolescence and user attrition. Next, we will increase the complexity of our simulation by controlling the ways hosts are connected within a zone, not just the inputs and outputs of the zone. Third, we will consider a heterogeneous environment with multiple platforms (e.g. various versions of Windows, MacOS and Linux and peripherals), each of which has an independent probability that an exploit exists. Finally, we currently use probability in the simulation and mathematical model, but in the future we will use a more complicated function that allows these artifacts to predict the performance of CZD with more sophisticated communication controls.

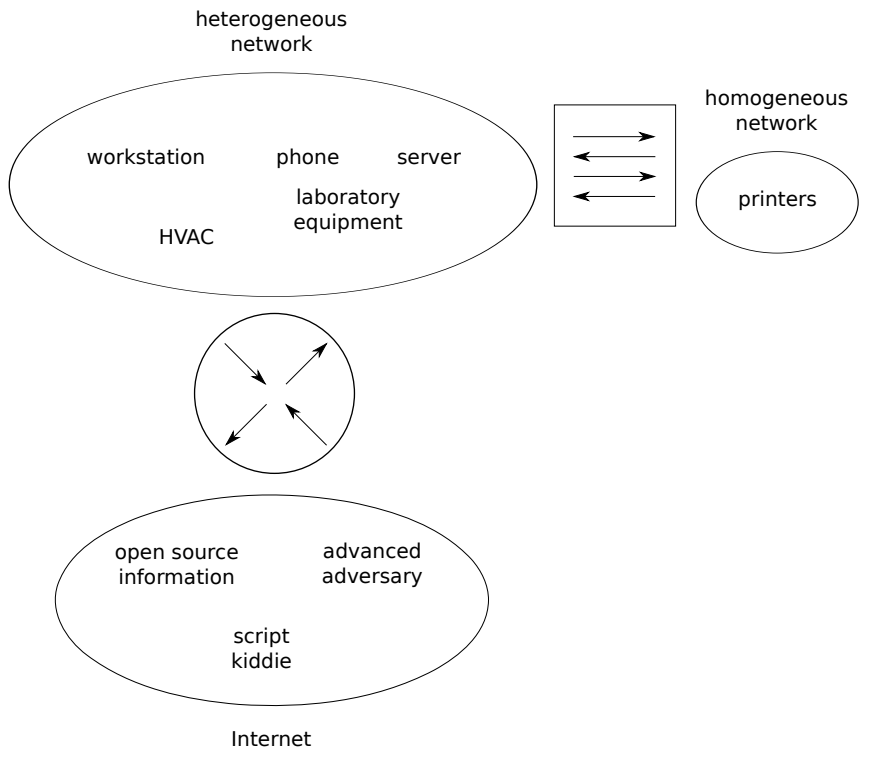

Fig. 10. Isolated printer zone.

In addition to these simulation improvements, we consider four major threads to continue this line of investigation: First, our attack model assumes that the isolation mechanism is completely secure. However, an especially sophisticated attacker could accomplish lateral movement and/or exfiltration within the constraints of the isolation technique. We will extend our artifacts to accommodate this more advanced threat which will encompass different brands of malware. The second thread of our continuation will be to instrument an emulation that will allow the system manager to validate individual configurations in a high fidelity virtualization of their subject. It appears that there is no optimal zone size: smaller is more secure, so the most secure zone size is one. However, it is not reasonable to put every host in their own zone. The system manager will use our simulation and emulation to decide on a reasonable size and use this to make a business decision to trade level of security for workplace productivity. Threads three and four will study means for populating zones that are sufficiently large to allow productivity. Our third thread will be to pursue dynamic zones which will constantly reconfigure to provide zones of a reasonable size with the most effective memberships. Dynamic zones can be considered a moving target defense, which is an added benefit. Finally, we believe organizing zones by device type is an effective zoning strategy.

The problem with the black box concept is a practical one: how to limit internal and external communication in a general purpose network. Flat, heterogeneous networks generally contain many types of devices, such as workstations and printers. It is impossible, or at least impractical, to limit all of the interconnections necessary to maintain a functioning network. However, grouping similar device types and work functions reduces the complexity to a manageable level. Logical, homogeneous networks, which we call Isolation Zones (IZs), can be readily firewalled to and from their physical, heterogeneous counterparts. IZs allow only essential communication and approximate a black box. To establish IZs, Open Systems Interconnection (OSI) layer two separation between devices must be guaranteed. Implementation options for this include private virtual local area network (VLAN) and Forced Forwarding (RFC 4562) which effectively create a second concentric IZ of size one within an IZ. The upstream router and firewall controls what each device can see at OSI layer three. For instance, our isolated printer zone (IPZ) denies incoming clear-text hypertext transfer protocol (HTTP), thus protecting administrative passwords from being revealed. It also denies most outgoing connections, except well protected services like Dynamic Host Configuration Protocol (DHCP), which denies C2 and data exfiltration channels. Figure 10 illustrates the IPZ concept. Another IZ approach is an isolated browsing zone (IBZ). Browsing the Internet provides malware access into the internal network. The outgoing connection also provides a conduit for malware $\mathrm{C} 2$ and data exfiltration. The browsers function as follows: A user asks for a web page, gets raw data and renders it into visual form. Raw data may or may not contain malware. Humans consume the rendered, not the raw data. Therefore, browsing can be done indirectly or remotely. IBZ doesn't care if data is good or bad, it isolates everything; it protects the internal network without sacrificing functionality. We imagine a number of other IZ techniques: Training zones comprise platforms for training. Facility zones network heating, ventilating and air conditioning (HVAC), fire control and supervisory control and data acquisition (SCADA) systems. Laboratory zones host microscopes and other experimental equipment. Guest zones provide visitors with Internet access. Remediation zones isolate vulnerable devices until they receive patches. Desktop zones allow only authenticated services. Server zones block the Internet and have limited internal access.

In this paper we proposed a simulation that allows the system manager or architect to predict the security of system configurations and a mathematical model that allows them to verify the simulation results. The modest quantitative results of this study lay the foundation for future models and zoning 
approaches that will approach the sophistication required to apply to modern networks. Our results in this paper are intuitive. The simulations in our future work will increase in size and complexity, and we anticipate the corresponding increase in fidelity will cause interesting properties to emerge.

\section{REFERENCES}

[1] R. Armstrong, J. Mayo, and Frank Siebenlist. Complexity science challenges in cybersecurity. Sandia National Laboratories SAND Report, 2009.

[2] Chee-Sing Chan. Complexity the Worst Enemy of Security. Computer World Hong Kong, December 2012.

[3] W. A. Conklin. State Based Network Isolation for Critical Infrastructure Systems Security. In 48th Hawaii International Conference on System Sciences, pages 2280-2287, Kauai, HI, USA, January 2015.

[4] Victor T. Costa and Luís Henrique M. K. Costa. Vulnerabilities and solutions for isolation in FlowVisor-based virtual network environments. Journal of Internet Services and Applications, 6(1), 2015.

[5] Iñigo Cuiñas, Paula Gómez, Manuel García Sánchez, and Ana Vázquez Alejos. Using vegetation barriers to improving wireless network isolation and security. In e-Business and Telecommunications, pages 428-438. 2009.

[6] Natalia C. Fernandes, Marcelo D. D. Moreira, Igor M. Moraes, Lyno Henrique G. Ferraz, Rodrigo S. Couto, Hugo E. T. Carvalho, Miguel Elias M. Campista, Luís Henrique M. K. Costa, and Otto Carlos M. B Duarte. Virtual networks: isolation, performance, and trends. Annals of telecommunications - Annales des télécommunications, 66(5-6):339$355,2011$.

[7] Lei Gong, Yong Zhao, and Jianhua Liao. Research on the Application Security Isolation Model. In Xuejia Lai, Dawu Gu, Bo Jin, Yongquan Wang, and Hui Li, editors, Forensics in Telecommunications, Information, and Multimedia, volume 56 of Lecture Notes of the Institute for Computer Sciences, Social Informatics and Telecommunications Engineering, pages 294-300. 2011.

[8] LanSheng Han, Ming Liu, and Zhixiang Liu. Type Based Network Isolation Against Net Virus. In 4th International Conference on Wireless Communications, Networking and Mobile Computing, pages 1-4, Dalian, China, October 2008.

[9] Mehdi Khoury, Seth Bullock, Gaihua Fu, and Richard Dawson. Improving measures of topological robustness in networks of networks and suggestion of a novel way to counter both failure propagation and isolation. Infrastructure Complexity, 2(1), 2015.

[10] Yeu-Pong Lai and Jui-Heng Tai. Network Security Improvement with Isolation Implementation Based on ISO-17799 Standard. In Tomoya Enokido, Leonard Barolli, and Makoto Takizawa, editors, NetworkBased Information Systems, volume 4658 of Lecture Notes in Computer Science, pages 69-78. 2007.

[11] Hongliang Liang, Changyao Han, Daijie Zhang, and Dongyang Wu. A Lightweight Security Isolation Approach for Virtual Machines Deployment. In Dongdai Lin, Moti Yung, and Jianying Zhou, editors, Information Security and Cryptology, volume 8957 of Lecture Notes in Computer Science, pages 516-529. 2015.

[12] Jiaqiang Liu, Yong Li, Huandong Wang, Depeng Jin, Li Su, Lieguang Zeng, and Thanos Vasilakos. Leveraging software-defined networking for security policy enforcement. Information Sciences, 327:288-299, 2016.

[13] R. V. Nunes, R. L. Pontes, and D. Guedes. Virtualized network isolation using Software Defined Networks. In IEEE 38th Conference on Local Computer Networks, pages 683-686, Sydney, Australia, October 2013.

[14] Paul Sery. Isolation Zones: Building Security into Network Architecture. In National Laboratories Information Technology Summit, Seattle, WA, USA, May 2015.

[15] Cao Wantian and Jiang Chengzhi. Intelligent communication network research of isolation security model based on the anomaly detection. In 2nd International Conference on Information Technology and Electronic Commerce, pages 167-171, Dalian, China, December 2014.

[16] Z. Yu, R. Subramanian, and S. Ahluwalia. Innovative and effective methodology for implementing network isolation for manufacturing environments. In IEEE International Symposium on Semiconductor Manufacturing, pages 365-368, San Jose, CA, USA, September 2005. 